\title{
Synthesis of the Low-Frequency Vibration Exciter
}

\author{
Dmitriy Fedorov \\ Department of Electric Coupling and \\ Automation Systems \\ Pskov State University \\ Pskov, Russia \\ dmitriy-fedorov-2012@mail.ru
}

Yulia Domracheva

Department of Electric Coupling and

Automation Systems

Pskov State University

Pskov, Russia

juli-politeh@yandex.ru

\author{
Andrei Khitrov \\ Department of Electric Coupling and \\ Automation Systems \\ Pskov State University \\ Pskov, Russia \\ khitrov.aa@gmail.com
}

\author{
Oksana Kozyreva \\ Department of Electric Coupling and \\ Automation Systems \\ Pskov State University \\ Pskov, Russia \\ ks_33n@mail.ru
}

\author{
Evgeniy Veselkov \\ Department of Electric Coupling and \\ Automation Systems \\ Pskov State University \\ Pskov, Russia \\ wes_el@bk.ru
}

\begin{abstract}
The article is devoted to synthesis of the lowfrequency vibration exciter for checking of sensors of acceleration. In many areas of the modern equipment sensors of acceleration working in very low range of frequencies are widely used. For checking and graduation of such sensors the vibration exciter capable to provide rectilinear horizontal harmonic oscillations of the calibrated accelerometer in so low range of frequencies are required. Low frequencies of fluctuations cause the necessity of creation of big amplitudes of movements for ensuring the acceptable values of amplitudes of accelerations. The low-frequency electrodynamic vibration exciter with a magnetic suspension of mobile part which is a component of the National Standard of the vibration movement of the Russian Federation is so far created. However, development of the modern equipment demands expansion of frequency ranges to the area of ultralow frequencies. One of requirements shown to the vibration exciter working in the ultralow range of frequencies is increase in amplitude of horizontal movements of a mobile part as with small amplitudes the speed and acceleration of the harmonious law of the movements proportional according to the frequency of fluctuations and a square of this frequency, will have small amplitude values. One of problems of realization of a control system of the electric drive of the vibration exciter consists in that a mobile part possesses indifferent position of balance. The centre of fluctuations of a mobile part is not defined and can be in any point on magnetic conductor length. That fluctuations had the steady centre in an average point of a magnetic conductor without use of a mechanical spring, the drive is supplied with an additional control system of fluctuations, or a so-called electromagnetic spring.
\end{abstract}

Keywords - vibration, vibration exciter, active magnetic suspension, digital control system, magnetization, electromagnetic drive.

\section{INTRODUCTION}

In many areas of the modern equipment (seismology, the space and aviation industry, high-powered power stations, construction of altitudinal objects, transport) sensors of acceleration (accelerometers) working in very low range of frequencies are widely used $(0,01 \ldots 5 \mathrm{~Hz})$. For checking and graduation of such sensors the vibrating exciters capable to provide rectilinear horizontal harmonic oscillations of the calibrated accelerometer in so low range of frequencies are required.

Low frequencies of fluctuations cause the necessity of creation of big amplitudes of movements for ensuring the acceptable values of amplitudes of accelerations. The lowfrequency electrodynamic vibrating [1] exciter with a magnetic suspension of mobile part [2] with a range of frequencies of $0,1-5 \mathrm{~Hz}$ and with an amplitude of fluctuations of $0,5 \mathrm{~m}$ which is a component of the National Standard of the vibration movement of the Russian Federation is so far created. However, development of the modern equipment demands expansion of frequency ranges to the area of ultralow frequencies to $0,01 \mathrm{~Hz}$.

This article examines the method of determining the traction force of the vibrating exciter, the synthesis of the digital control system and the results of the experiment in physical modeling of the vibrating exciter. 


\section{MATERIALS AND METHODS}

Driving part of the vibrating stand (fig. 1) consists of a magnetic conductor, four motionless coils of magnetization 1 and one mobile coil 2. The magnetic conductor consists of five horizontally located ferromagnetic cores of round cross section - three longitudinal 3 and two cross 4 , connecting face surfaces of the longitudinal cores.

Coils of magnetization are put on cross cores, connected consistently, are powered a direct current $\mathrm{I}_{1}$ from the power supply unit and create constant magnetic field on all length of a working air gap between central and side longitudinal cores. The mobile coil is put freely on the central longitudinal core. At power supply of the coil with the sinusoidal setting current it will be affected in the longitudinal direction by the harmonious force of electrodynamic influence.

The mobile coil together with the examinee accelerometer forms a mobile part of the vibrating stand which is supplied with system of an active magnetic suspension [3].

The active magnetic suspension, thanks to lack of lubricant and mechanical contact between mobile and motionless parts, allows to exclude influence of friction and dust on quality of working off of the setting signal.

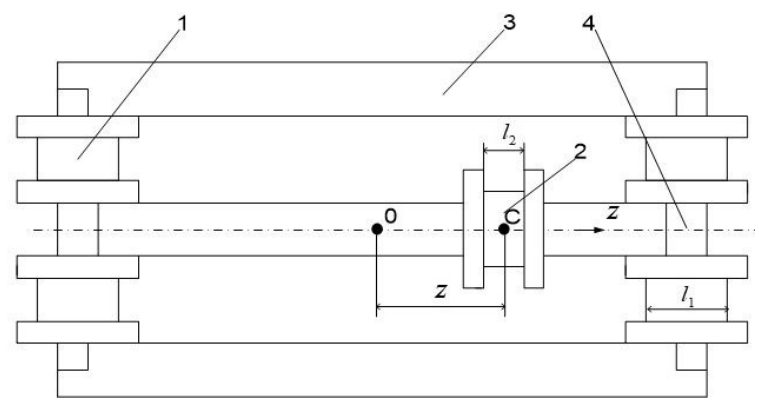

Fig. 1. System of the electrodynamic drive of a vibrating exciter.

To synthesize the parameters of a low-frequency vibration exciter, it is necessary to obtain an analytical expression for the traction force of the mobile part. Given the large linear size of the exciter, we scheme it as an electromagnetic system with distributed parameters.

Let $\mathrm{z}$ be the coordinate of the center of the moving coil, measured from the middle of the length of the magnetic circuit.

Having written down expression for magnetic energy [4] systems

$$
w=\frac{1}{2} L_{11} i_{1}^{2}+\frac{1}{2} L_{22} i_{2}^{2}+L_{12} i_{1} i_{2},
$$

and taking the partial derivative of it with respect to the $\mathrm{z}$ coordinate, considering that self-inductances $\mathrm{L}_{11}$ and $\mathrm{L}_{22}$ do not depend on displacement, we arrive at an expression for the electromagnetic traction force of the form

$$
\mathrm{P}_{\mathrm{em}}=\frac{\partial \mathrm{L}_{12}}{\partial \mathrm{z}} \mathrm{I}_{1} \mathrm{i}_{2}(\mathrm{t})
$$

where I1 is the current of the magnetizing coils (primary circuit); i2(t) - current of the moving coil (second circuit); L12 - coefficient of mutual inductance of these circuits.

The mutual inductance of the circuits can be defined as

$$
\mathrm{L}_{12}=\mathrm{L}_{21}=\Psi_{21} / \mathrm{I}_{1}^{\prime}
$$

where $\Psi_{21}$ is the flux linkage of the flows of the coils of the first circuit with the turns $\mathrm{w}_{2}$ of the second circuit.

It is supposed that the magnetic system is not saturated both linear and, therefore, the magnetic resistance of steel is constant. Therefore, we will use the principle of superposition. Proceeding from it, we decompose the magnetic system into two subsystems, one of which is powered by the left magnetizing coils, the other - by the right ones. We replace the magnetodriving force created by two coils of magnetization located on a cross core with one, located at the beginning of a longitudinal core.
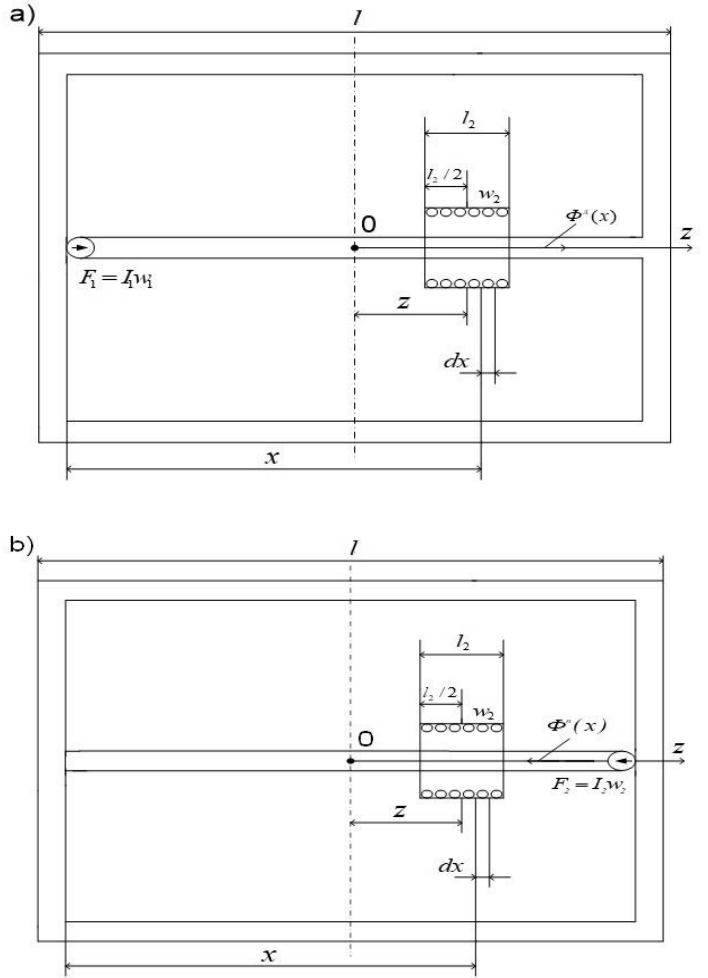

Fig. 2. Decomposition of the magnetic system into two subsystems powered by magnetizing coils a) left; b) right.

In the moving coil, we select an element with a length $\mathrm{dx}$ at a distance $\mathrm{x}$ from the left end of the longitudinal rods of the magnetic circuit. The element has the number of turns $d w=\frac{w_{2}}{l_{2}} d x$, where $l_{2}$ is the length of the winding of the moving coil.

In this case, it is obvious that the component of the magnetic induction in the direction of the $\mathrm{z}$-axis is in the air $B_{z}^{B}=0$, and only the radial component $B_{r}^{B} \neq 0$ is present. In addition, let the diameter $\mathrm{l}_{1}$ of the magnetizing coils be 
Environment. Technology. Resources. Rezekne, Latvia Proceedings of the $13^{\text {th }}$ International Scientific and Practical Conference. Volume 3, 66-71

small in comparison with the length 1 of the magnetic circuit.

We consider that the left and right magnetizing coils create counter-directed magnetic fluxes.

Let's find the magnetic fluxes created by the left and right coils.

The considered magnetic system is the magnetic line with the distributed parameters consisting of the longitudinal and cross magnetic resistances distributed on length. In this case, longitudinal magnetic resistance is magnetic resistance of ferromagnetic cores (steel), and cross - magnetic resistance of an air gap between central and side cores. This magnetic line can be considered uniform, since all the longitudinal resistances of the sections of the cores of the same length are equal to each other, and the cross resistances of the sections of the line of the same length, due to the parallelism of the cores, are also equal to each other.

We will neglect the magnetic resistances of the end cores. The settlement scheme of the magnetic circuit for the case with left magnetizing coils is shown in Fig. 3. The diagram of the magnetic circuit for the case with the righ coils has a similar view.

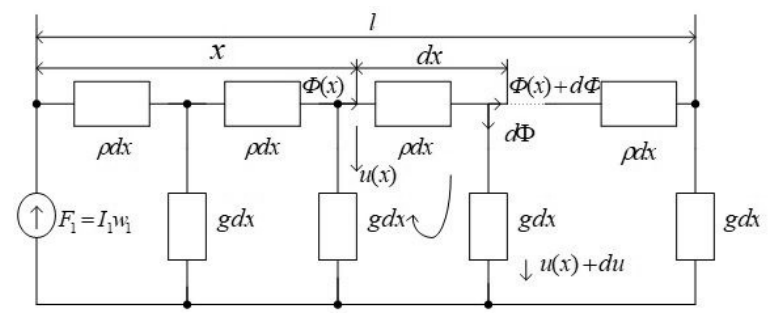

Fig. 3. The settlement scheme of the magnetic circuit for the case with left magnetizing coils.

On the provided scheme: $\mathrm{dx}$ - infinitesimal element of the line length; $\rho$ - reduced longitudinal magnetic resistance per unit length of the magnetic circuit; g reduced cross magnetic conductivity of a unit of line length; $\Phi(\mathrm{x})$ - magnetic flux; $\mathrm{u}(\mathrm{x})$ - magnetic voltage; $\mathrm{F}(\mathrm{x})=\mathrm{I}_{1} \mathrm{w}_{1}$ - magnetodriving force created by the left magnetizing coils.

From the scheme according to Kirchhoff's laws, we have the following equations:

$$
\begin{aligned}
& -d u / d x=\rho \Phi(x) ; \\
& -d \Phi / d x=g u(x) .
\end{aligned}
$$

At the solution of the equations [5] it is necessary to set boundary conditions. They are known only for $\mathrm{u}$ and have an appearance $\mathrm{u}=\mathrm{F}_{1}$ at $\mathrm{x}=0$; $\mathrm{u}=0$ at $\mathrm{x}=\mathrm{l}$. follows

From the above, the following differential equation

$$
\frac{d^{2} u}{d x^{2}}-a^{2} u=0
$$

where $a=\sqrt{g \rho}=\frac{\sqrt{G^{\mathrm{IP}} R^{\mathrm{IP}}}}{l}$.

The solution of this equation is the expression

$$
\mathrm{u}^{\mathrm{J}}=\mathrm{F}_{1}[\operatorname{ch}(\mathrm{ax})-\mathrm{cth}(\mathrm{al}) \cdot \operatorname{sh}(\mathrm{ax})]
$$

Having made a number of substitutions, we obtain the following expression for the traction force of the electrodynamic vibration exciter:

$$
\begin{gathered}
\mathrm{P}_{\mathrm{em}}=\frac{\partial \mathrm{L}_{12}}{\partial \mathrm{z}} \mathrm{I}_{1} \mathrm{i}_{2}(\mathrm{t})= \\
=\frac{\mathrm{F}_{1} \mathrm{~F}_{2}(\mathrm{t}) \mathrm{g}}{\operatorname{ch}\left(\frac{\mathrm{al}}{2}\right)}\left(1+\frac{1}{2} \mathrm{a}^{2} \mathrm{z}^{2}\right) .
\end{gathered}
$$

From the obtained expression it follows that the traction force will be purely harmonic at $\mathrm{F}_{2}(\mathrm{t})=\mathrm{w}_{2} \mathrm{I}_{2} \sin \omega \mathrm{t}$, if $\mathrm{a}=\sqrt{\mathrm{g} \rho}=0$. As at $\mathrm{g}=0$ we have $\mathrm{Pe}=0$, the necessary condition takes a form $\rho=0$.

But, since even with small inductions $\rho \neq 0$, the presence of higher harmonics is inevitable in the expression for the force. They can be reduced by avoiding saturation of the steel. A recommendation has been developed to ensure a given permissible level of higher harmonics.

The synthesis of the parameters of the vibration exciter is carried out taking into account the expression for the traction force. Since when using the full number of turns of the coils with a decrease in the oscillation frequency, the currents in the coils decrease and take values of the same order of magnitude with the noise currents, it is necessary to increase the values of the currents in the windings.

This can be achieved by sectioning the windings of the moving and stationary coils, adjusting the number of turns in them with constant currents. The value of the currents can be set based on the maximum vibration frequency of the moving part, which will correspond to the maximum number of turns in the coils. The magnetodriving forces will be regulated with constant currents in windings. At a given frequency range change of traction effort by 400 times is observed. In this case, the number of turns of primary and secondary coils at the boundaries of the frequency range differ by 7,5 and 53 times respectively.

Next problem of synthesis of the low-frequency vibration exciter consists in that a mobile part possesses indifferent position of balance. The centre of fluctuations of a mobile part is not defined and can be in any point on magnetic conductor length. That fluctuations had the steady centre in an average point of a magnetic conductor without use of a mechanical spring, the drive is supplied with an additional control system of fluctuations, or a socalled electromagnetic spring.

In case of realization of control systems of the movement in analog execution, the regulator will have constant control (in sense of constancy of coefficients of strengthening of feedback). However, setup of the regulator has to change with change of frequency of fluctuations. As 
the set range of working frequencies assumes change of frequency by 20 times, use of the analog regulator will be difficult. This circumstance caused transition to digital control [6] which advantage is flexibility.

The object of control is the moving part of the vibration exciter, which is a dynamic link, the input (control) variable of which is the current in the moving coil of the magnetic circuit, and the output (controlled) variable is the coordinate of the center $C$ of the moving coil relative to the center of oscillations $O$. Considering that an active magnetic suspension is used the moving part of the vibration exciter, there is no friction force and the equation of the object has the form

$$
\begin{aligned}
& m \ddot{x}(t)=F(t) ; \\
& F(t)=h_{2}(t),
\end{aligned}
$$

where h - coefficient of transfer "current-force", depending on the current I1 in the magnetizing coils; $\mathrm{m}$ - the mass of the moving part.

The resulting discrete model of the control object in the form of difference equations:

$$
\begin{gathered}
\mathrm{y}(\mathrm{k}+1)=\mathrm{Ay}(\mathrm{k})+\mathrm{Bi}_{2}(\mathrm{k}) ; \\
\mathrm{z}(\mathrm{k})=\mathrm{Cy}(\mathrm{k}),
\end{gathered}
$$

where $y(k)=\begin{aligned} & z(k) \\ & \dot{z}(k)\end{aligned} ; y(k+1)=\begin{aligned} & z(k+1) \\ & \dot{z}(k+1)\end{aligned} ; A=$

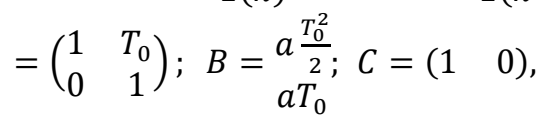

where $\mathrm{k}$ is an integer; $\mathrm{T0}$ - period of quantization of an ideal quantizer; $\mathrm{a}=\mathrm{h} / \mathrm{m}$ - coefficient.

The drive control system consists of a displacement sensor $\delta(z)$ of the movable part in the longitudinal direction from the center of vibrations and a regulator. The signal from the sensor is processed by the regulator, amplified and summed up with the reference current.

The signal from the displacement sensor goes to the input of the transducer located in the control unit. The differential displacement sensor, as one of the options, consists of two ferromagnetic coils moving together with the moving part of the vibration exciter along opposite sides of the tensioned electrical steel strip (Fig. 4).

This realization allows to achieve almost linear dependence of the measured voltage on the position of an object. Small nonlinearity, connected with inhomogeneity of material of the stretched tape, are eliminated by introducing second-order digital filter into the control system according to the control signal.

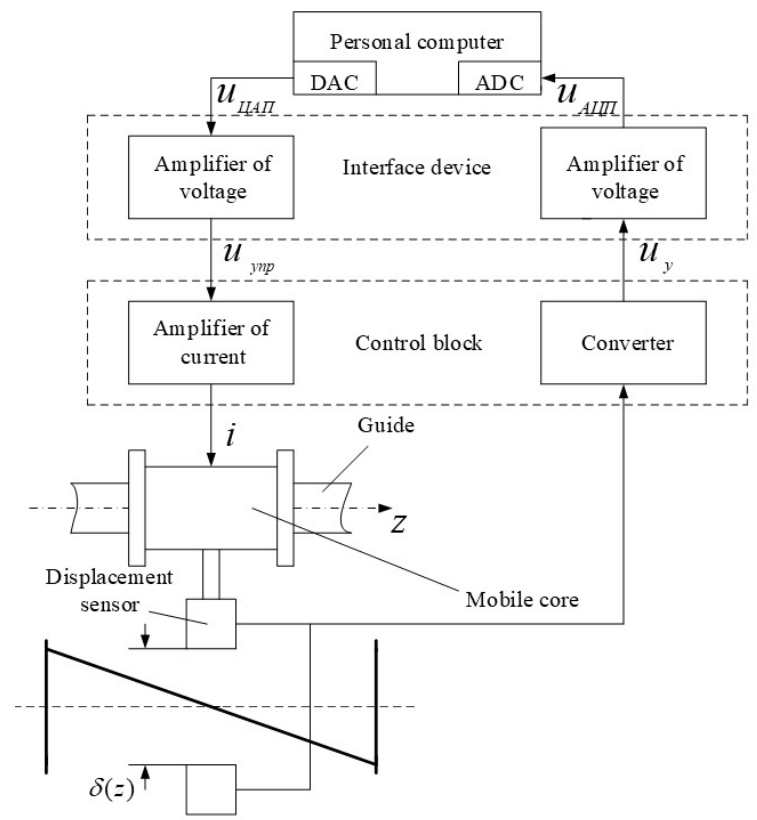

Fig. 4. Function chart of a control system.

The output voltage of the converter is proportional to the position of the moving part of the vibration exciter. This voltage through a matching amplifier located in the interface device is fed to the input of the analog-to-digital converter of the input-output board of the personal computer.

A personal computer acts as a control device and implements a digital controller for object control. The signal calculated by the regulator [7] is fed to the input of the digital-to-analog converter of the input-output board. The output voltage through the matching amplifier of the interface device is fed to the input of the current amplifier.

The current amplifier controls the current i2 of the moving coil of the vibration exciter in proportion to the voltage supplied to the input.

A filter can be added to the system output to smooth out micro current surges and acceleration.

\section{RESULTS AND DISCUSSION}

When carrying out measurements, the problem of experimental verification of the main theoretical relations obtained when solving the problem of synthesis of a lowfrequency electrodynamic vibration exciter was solved.

Experimental studies were carried out on a model of a vibration exciter created at the Pskov State Polytechnic Institute

The model has the following parameters:

- amplitude of fluctuations at a frequency of $1 \mathrm{~Hz}$ is equal to $0,125 \mathrm{~m}$;

- the magnetic conductor is made of Steel 10, length of longitudinal cores of $0,66 \mathrm{~m}$, cross cores $0,42 \mathrm{~m}$, diameter of cores of 0,1 m, an air gap between longitudinal cores of $0,06 \mathrm{~m}$; 
- constant magnetic field in air gaps between longitudinal cores is created by means of four magnetizing coils, the $0,1 \mathrm{~m}$ coil radius, the $0,06 \mathrm{~m}$ coil length, a framework of the coil is made of textolite, thickness of walls of $5 \mathrm{~mm}$, coils have on 530 turns of a copper wire diameter of $1,55 \mathrm{~mm}$;

- the mobile coil has the $74 \mathrm{~mm}$ radius, a framework from textolite, the $0,05 \mathrm{~m}$ coil length, a gap between the coil and the central core of a magnetic conductor along which it moves of $8 \mathrm{~mm}$, number of turns is 350, a winding is executed by an aluminum wire; diameter of a wire of 1 $\mathrm{mm}$.

- the mobile coil has the electromagnetic suspension consisting of four linear active magnetic support moving along a cylindrical guide from Steel 3 diameter of $25 \mathrm{~mm}$, and the linear active magnetic support limiting the rotary pendular movements in the direction;

To measure the traction force, a stop was installed on the central core of the magnetic circuit. When the currents of the moving and stationary coils interact, a force arises that presses the moving coil against the stop. A thread thrown over a block was attached to a moving coil; a cup with a weight was suspended from the second end of the thread.

If the current is gradually reduced from a certain maximum value $(1,4 \mathrm{~A})$ to the value at which the coil will detach from the stop, then we can assume that $\mathrm{P}_{\mathrm{em}}=\mathrm{mg}$, where $\mathrm{m}$ is the mass of the cup with the load. The traction force was measured by measuring the breakout force. $\mathrm{I} 1=4 \mathrm{~A}$ current was supplied to the stationary coils, and I2 current in the interval $(0,16 \div 1,4) \mathrm{A}$ to the moving one.

Thus, the dependence Рем(I2) was removed at fixed values of the $\mathrm{z}$ coordinate and current I1. The form of the function and the theoretical curve at $\mathrm{z}=125 \mathrm{~mm}$ and $\mathrm{I} 1=4 \mathrm{~A}$ are shown in Fig.5.

Fig. 5 shows that the dependence is linear, while the calculated value of the traction force is $8-12 \%$ higher than the experimental one.

The reason for the discrepancy may be unaccounted for in the calculations such factors as the magnetic resistance of the joints of the longitudinal and transverse rods of the magnetic circuit; inhomogeneity of the magnetic properties of the material along the length of the rods; leakage fluxes of magnetizing coils.

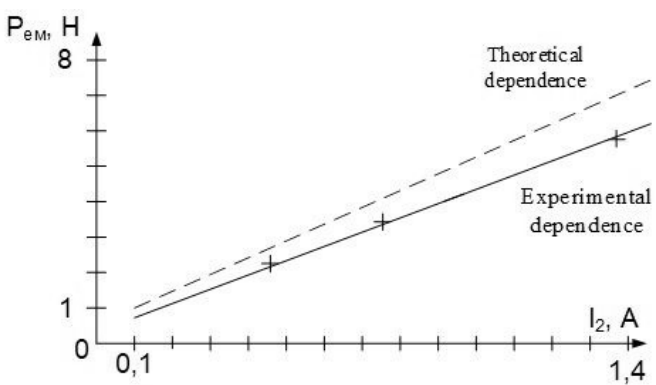

Fig. 5. Experimental and theoretical dependences of the traction force on the current of the moving coil.
In a similar way, the dependence Pem(z) was removed at fixed values of $\mathrm{I} 1=4 \mathrm{~A}$ and $\mathrm{I} 2=0,4 \mathrm{~A}$. The form of the function and the theoretical curve are shown in Fig.6.

Fig. 6 shows that the experimental dependence of the traction force on the coordinate has a parabolic form, which qualitatively confirms the theory. The theoretical curve shown in this figure corresponds to the second term in the expression for the thrust force. It follows from the comparison of the curves that the experimental dependence of the traction force on the coordinate is stronger than the theoretical one.

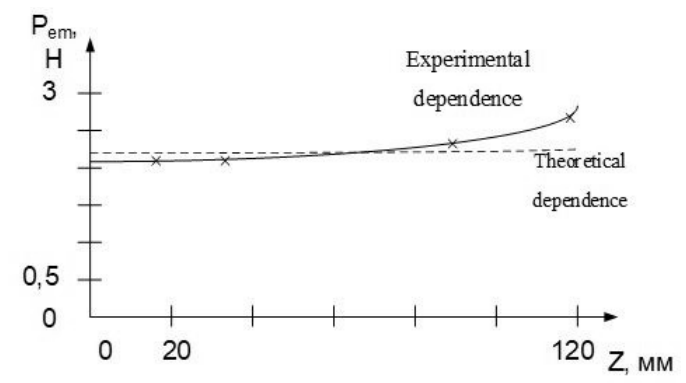

Fig. 6. Dependence of the traction force on the coordinate of the moving part.

For the given values of the currents $\mathrm{I} 1=4 \mathrm{~A}$ and $\mathrm{I} 2=0,4 \mathrm{~A}$, the contribution of the second term according to the experiment is $19 \%$, while according to the theoretical dependence at the same currents this contribution is $0.9 \%$. Air gaps in joints of longitudinal and cross cores which were not considered when calculating a magnetic conductor are the reason of divergences. In addition, the magnitude of the traction force can be influenced by the inhomogeneity of the magnetic field in the working gap in the direction of the coordinate $\mathrm{z}$ where the moving coil moves.

The available equipment made it possible to perform harmonic analysis in amplitude and acceleration and measure the harmonic distortion, starting from $2 \mathrm{~Hz}$. The measurements were carried out at a frequency of $2.05 \mathrm{~Hz}$. The amplitude harmonic coefficient was found to be $0.2 \%$, although spikes of up to $0.7 \%$ were occasionally observed. The acceleration harmonic coefficient did not exceed $1.7 \%$.

\section{CONCLUSIONS}

The article presents the main problems associated with the synthesis of a low-frequency vibration exciter. A method for determining the traction force of a lowfrequency vibration exciter with an active magnetic suspension of the moving part is presented. The problem associated with the uncertainty of the position of the moving part of the vibration exciter is considered.

As a solution to this problem, a digital control system for a low-frequency vibration exciter has been proposed and implemented. The results of experimental studies on the physical model of the vibration exciter are presented. A comparative analysis of theoretical and experimental dependences is carried out. The results of the experiment 
qualitatively and quantitatively confirm the theoretical researches.

The research results are implemented at the Mendeleev Research Institute.

\section{ACKNOWLEGMENTS}

This article is prepared with partial help of Yury Zhuravlev. We also thank the Pskov Polytechnic Institute, which provided an opportunity for experimental research. Special thanks to the Mendeleev Research Institute.

\section{REFERENCES}

[1] M. D. Genkin, Electrodynamic vibrators. M.: Mechanical engineering, 1975. - $98 \mathrm{p}$.
[2] A. N. Gribov, Y. N. Zhuravlev, V. G. Matsevich. The vibrating stand of low-frequency vibration with an active magnetic suspension of a mobile part//Modern problems of improvement of measuring instruments of mechanical sizes. L.: Energoatomizdat, 1986. P. 7782.

[3] Y. N. Zhuravlev, Active magnetic bearings: Theory, design, applications. SPb.: Polytechnics, 2003. - 206 p.

[4] D. B. Sivukhin, General course of physics. Vol. III. Electricity. Prod. the 4th, stereotypic. M.: MIPT publishing house, 2004. - 656 p.

[5] B. K. Bul, fundamentals of theory and calculation of magnetic fields. M. - L.: Energy, 1964. - 464 p.

[6] B. Kuo, Theory and design of digital control systems. M.: Mechanical engineering, 1986. - $447 \mathrm{p}$.

[7] G. Olson, D. Piani, Digital systems of automation and management. SPb.: Nevsky publishing house dialect, 2001. -557 p. 